\title{
Ansiedade social: gênero, orientação sexual e classe social
}

\author{
Social anxiety: gender, sexual orientation and social class
}

Ansiedad social: género, orientación sexual y clase social

\author{
Mozer de Miranda Ramos*, Sophia Helena Rito** , \\ Elder Cerqueira-Santos***
}

\section{RESUMO}

A presença de desigualdades hierárquicas expõe minorias soPalabras clave: ciais a uma maior experimentação de estresse, sofrendo interferências em sua vida e saúde. $\mathrm{O}$ objetivo deste estudo foi investigar a relação entre o pertencimento a grupos minoritários e a presença de sintomas de ansiedade social. Participaram 1049 pessoas, com idades entre 18 e 56 anos ( $\mathrm{M}=25,98$; DP = 7,56), $59,8 \%$ homens. Os resultados indicaram que pertencer a algumas minorias sociais, como ser mulher, não-heterossexual ou dispor de menor renda é um preditor de ansiedade social. Em indivíduos não-heterossexuais, a presença de homofobia internalizada demonstrou ampliar a ocorrência de sintomas de TAS (Transtorno de Ansiedade Social). O estudo demonstrou que a experimentação do estresse social por grupos minoritários apresenta relação com desfechos negativos em saúde mental.

\section{ABSTRACT}

The presence of hierarchical inequalities exposes social minoriKeywords: mental ties to higher levels of stress, which can interfere in their qualihealth, social ty of life and health. The objective of this study is to investigate anxiety, sexuality, the relationship between belonging to minority groups and the minorities. presence of symptoms of social anxiety. Participation included saúde mental, ansiedade social, sexualidade, minorias.

\footnotetext{
* Mestre e doutorando em psicologia, Universidade Federal de Sergipe, Aracaju. ORCID: https://orcid.org/0000-0001-5164-1543. Contacto: mozeramos@yahoo.com.br ** Graduanda em Psicologia, Universidade Federal de Sergipe, Aracaju.

*** Doutor em Psicologia, Universidade Federal de Sergipe, Aracaju. ORCID: https:// orcid.org/0000-0003-1116-6391
} 
1,049 people, between the ages of 18 and 56 years $(M=25.98$; SD $=7.56$ ), including $59.8 \%$ men. The results indicate that belonging to a social minority, for example, as a woman, non-heterosexual or low-income, is a predictor of social anxiety. Among non-heterosexual individuals, the presence of internalized homophobia was seen to increase the occurrence of symptoms of social anxiety disorder. The study showed that the social stress experienced by minority groups is associated with negative effects on mental health.

\section{RESUMEN}

La presencia de desigualdades jerárquicas expone a las mino-

Palavras-chave: rías sociales a una mayor experiencia de estrés, lo que genera interferencias en su vida y salud. El objetivo de este estudio fue investigar la relación entre la pertenencia a grupos minoritarios y la presencia de síntomas de ansiedad social. Los participantes salud mental, ansiedad social, sexualidad, minorías. fueron 1049 personas, con edades comprendidas entre los $18 \mathrm{y}$ los 56 años ( $\mathrm{M}=25,98 ; \mathrm{SD}=7,56), 59,8 \%$ hombres. Los resultados indicaron que pertenecer a alguna minoría social, como ser mujer, no ser heterosexual o tener menores ingresos, es un predictor de la ansiedad social. En los individuos no heterosexuales, se demostró que la presencia de homofobia interiorizada amplifica la aparición de los síntomas del TAS (Trastorno de Ansiedad Social). El estudio demostró que la experiencia de estrés social de los grupos minoritarios está relacionada con resultados negativos en materia de salud mental. 


\section{Introdução}

A presença de desigualdades sociais atua como um forte preditor de iniquidades em saúde, o que incita disparidades referentes à prevalência de distress (Rocha, Perez, Rodriguez-Sanz, Borrel, \& Obiols, 2010; Silva \& Santana, 2012). Para algumas comunidades, as normas e tradições em um determinado contexto histórico-social estão relacionadas com a experimentação de sofrimento psíquico (Bilodeau, Marchand, \& Demers, 2020). Nessa direção, atos ou atitudes hostis direcionadas a grupos sociais específicos possuem um efeito acumulativo no decorrer do tempo, interferindo no comportamento dos indivíduos dos grupos que as sofrem (Assari, 2019; Lefevor, Janis, Franklin, \& Stone, 2019).

O principal referencial teórico em análises sobre a relação entre grupos minoritários e bem-estar psíquico é postulado pelo modelo de estresse social (Schwartz \& Meyer, 2010). Esses estudos buscam analisar a relação entre o sofrimento psicológico e as minorias (sexuais, inicialmente) (Meyer, 2003). Dentre os resultados encontrados, é apontado que pertencer a uma minoria, seja de classe, raça, gênero ou de orientação sexual incide na prevalência de desfechos negativos em saúde mental (Cohen, Blasey, Taylor, Weiss, \& Newman, 2016; McClain et al., 2016).

Atrelado a isso, minorias sexuais, como Lésbicas, Gays e Bissexuais (LGB) estão mais suscetíveis a sofrer as consequências do estresse minoritário, em decorrência do processo de estigmatização que estão submetidas. Possuindo, assim, maior possibilidade de apresentar transtornos psicológicos, como ansiedade e depressão (Alves, Paveltchuk, Carvalho, \& Falcone, 2017). Essa evidência pode ser explicada pelo Modelo do Estresse de Minoria desenvolvido por Meyer (1995), dado seu funcionamento imprescindível para o entendimento das condições vivenciadas por indivíduos LGB (Meyer, 2003). De acordo com o modelo, minorias sexuais estão mais expostas ao estresse oriundo de estigmas quando comparadas a indivíduos heterossexuais (Paveltchuk \& Borsa, 2020); apresentando, assim, maior disposição a apresentar problemas de saúde geral, resultado de uma maior exposição a eventos discriminatórios e aversivos de forma insistente (Frost, Levahot, \& Meyer, 2011; Meyer, 1995, 2003). Posteriormente, esse modelo foi ampliado para contemplar também minorias de gênero, como as pessoas trans e em não-conformidade de gênero (Hendricks \& Testa, 2012). 
A vivência de episódios de estresse social, como experimentação de eventos discriminatórios, é configurada como uma das principais fontes de estresse crônico, resultando em prejuízos na saúde física e emocional do indivíduo (Goto, Couto, \& Bastos 2013). Existem outros dois elementos considerados fundamentais para esse modelo: a ocultação da orientação sexual e a internalização da homofobia. A ocultação da orientação sexual está relacionada ao encobrimento da identidade sexual de si ou de outros, por conta do medo ou de uma representação social negativa acerca da diversidade sexual, por exemplo (Meyer, 2003). Quando indivíduos não-heterossexuais interiorizam essas estruturas, reprimindo-as ou as direcionando contra sua identidade sexual ou a de outros que destoem do padrão heteronormativo, é apontada a presença de homofobia internalizada (Antunes, 2017; Herek et al., 2009; Silveira \& Cerqueira-Santos, 2019).

A homofobia internalizada pode incidir como agente de danos psicológicos, derivando em sentimentos de insatisfação com aspectos do próprio indivíduo (Herek, Cogan, Gilis, \& Glunt, 1998). Fator que pode proporcionar isolamento social, insônia, depressão, ansiedade, sentimentos como o de insuficiência, medo, tristeza, angústia ou vergonha (Antunes, 2017). Essa atua, junto à ocultação da orientação e às experiências de vitimização, como reconhecidas preditoras de adoecimento psíquico na comunidade não-heterossexual e como os principais pilares da Teoria do Estresse de Minoria (Newcomb \& Mustanski, 2010; Walch, Ngamake, Bovornusvakool, \& Walker, 2016).

A Teoria do Estresse de Minoria é considerada interseccional no cenário atual, ou seja, ressalta que quanto mais sobreposições minoritárias um indivíduo pertencer, maior será a possibilidade de comprometimento do seu bem-estar (Paveltchuk \& Borsa, 2020). Nessa direção, o modelo proposto é definido como o conflito proveniente entre os indivíduos e suas experiências coletivas (Meyer, 2003; Meyer \& Frost, 2013) e é caracterizado como: (I) único - ou seja, aditivo aos estressores comumente experimentados por toda a sociedade; (II) crônico - pertinente às estruturas estabelecidas, e; (III) social - oriundo de processos institucionais, estruturais e individuais, ou condições taxativas (Meyer, 2003).

A vivência contínua de eventos discriminatórios, mesmo que de modo vicário, é seguida por comportamentos de insegurança frente às 
condutas sociais, além da adoção de uma postura de hipervigilância como forma de conduta protetiva (Riggle, Folberg, Richardson, \& Rostosky, 2021). Ambos estados são elementos estreitamente associados à manifestação do Transtorno de Ansiedade Social (TAS) (American Psychiatric Association, 2014; Ramos \& Cerqueira-Santos, 2021).

A sociedade atual está fundada em uma estrutura hierárquica, operacionalizada pelo machismo e pela misoginia, na qual as mulheres ocupam a base da sociedade, e sofrem repercussões negativas em diversos âmbitos (Connor, Glick, \& Fiske, 2016). Por exemplo, a crença de que para uma mulher ser completa deve alcançar a maternidade, bem como a vinculação do amor à sua aparência estética, e por conseguinte, o desenvolvimento de uma perspectiva irreal sobre si mesma e seu valor diante o mundo (Duarte \& Paulino, 2021). Não por coincidência, os homens que se aproximam do estereótipo feminino são associados a figuras de subordinação (Ramos \& Cerqueira-Santos, 2020). Diante disso, transgressões estereotipadas de gênero podem ser atribuídas por homens gays à rejeição sofrida, internalizando aversão a comportamentos afeminados (Juárez-Chávez, Cooney, Hidalgo, Sánchez, \& Poteat, 2018).

A respeito das disparidades de saúde, essas denotam maior complexidade quando há associação entre identidades minoritárias diferentes (Frost \& Meyer, 2012). Com efeito, mulheres lésbicas e bissexuais apresentam indicadores de saúde mental mais negativos do que mulheres heterossexuais (Koh \& Ross, 2006). Assim como mulheres de minorias étnicas; visto que uma mulher negra e lésbica enfrenta situações muito discrepantes das experimentadas por uma mulher branca e lésbica, em que cada cenário proposto acarreta um grau de estresse e ansiedade diferente (Frost \& Meyer, 2012). Além disso, conforme a disponibilidade de recursos econômicos identifica-se uma maior prevalência de problemas em saúde mental, com destaque também ao gênero feminino (Rocha et al., 2010).

Considerando-se que minorias sexuais precisam lidar com um maior contingente de discriminações no âmbito social, é compreensível que apresentem maior prevalência de sintomas ansiosos que pessoas heterossexuais (Cohen et al., 2016). Indivíduos com TAS identificam interações sociais como mais ameaçadoras comparados aos não ansiosos, principalmente os com menor abertura sobre sua orienta- 
ção sexual (Pachankis \& Goldfried, 2006). No estudo de Pachankis e Goldfried (2006), homens gays apresentaram maior ansiedade social e medo de avaliação negativa contrapostos a homens heterossexuais. Em concordância, Wadsworth e Skelton (2015) encontraram em seu estudo maiores níveis de ansiedade para indivíduos bissexuais quando comparados a heterossexuais, gays e lésbicas. A avaliação de indivíduos pertencentes a grupos minoritários quanto a sintomas de ansiedade social pode contribuir para o entendimento de como a vivência de estresse de minoria repercute negativamente na saúde mental de indivíduos.

Diante disso, o presente artigo se propôs a investigar a relação entre o pertencimento a grupos minoritários e a presença de sintomas de ansiedade social. Buscou-se analisar minorias sociais de vários grupos hierárquicos, como classe, raça/cor, gênero e orientação sexual, e suas diferentes interações nas evidências de ansiedade social. Ademais, propôs-se investigar a relação dos fatores direcionados à experimentação do estresse social em minorias sexuais, como a presença de homofobia internalizada e a interferência dos níveis de violência sofrida na prevalência do transtorno analisado.

\section{Método}

Esta é uma pesquisa transversal, de caráter quantitativo, e o nível de aprofundamento é descritivo exploratório. Foi realizado um levantamento online e a divulgação ocorreu através de redes sociais. A amostragem foi não probabilística, dada por conveniência.

\section{Participantes}

Participaram do estudo 1049 respondentes, moradores do Brasil, mais da metade composto pelo gênero masculino (59,8\%). A média de idade foi de 25,98 anos $(D P=7,56)$, com amplitude de 18 a 56 anos. Nessa amostra, $62,2 \%$ declararam ser heterossexuais, seguidos por $35,4 \%$ de lésbicas, gays ou bissexuais. Quanto ao status de relacionamento, $43,5 \%$ afirmaram estar solteiros(as). A média da idade da primeira relação sexual foi de 17,32 anos $(D P=3,14)$, com amplitude entre 7 e 34 anos. Metade da amostra se autodeclarou como branca, e o nível de escolaridade que predominou foi Ensino Superior Incompleto (43,2\%) seguido pelo Ensino Superior Completo (20,4\%). A distribuição geo- 
gráfica abarcou grande parte dos estados brasileiros. Desses, a maioria dos respondentes foi de Sergipe (32,3\%), São Paulo (14,6\%) e Bahia $(13,6 \%)$. Dentre os homens, $19,5 \%$ se declararam afeminados e $80,5 \%$ não-afeminados, entre os afeminados, 39,9\% são não-heterossexuais.

Com relação à renda domiciliar mensal, predominou os que vivem com menos de 2 salários mínimos mensais (31,9\%), as definições acerca da renda seguiram os critérios do IBGE no que preconiza classes $\mathrm{A}$, B, C, D e E com base nos salários mínimos mensais da família. Nesse estudo, incluiu-se também o recorte da classe E-, para os que vivem com até 1 salário mínimo. Dentre os indivíduos não-heterossexuais ( $n=371), 69,8 \%$ responderam já terem sofrido violência psicológica, $17,0 \%$ física, $27,0 \%$ sexual, 9,4\% financeira e $29,9 \%$ institucional pelo menos alguma vez no decorrer da vida por conta de sua orientação sexual. A caracterização completa dos participantes está disponível na Tabela 1.

Tabela 1

Caracterização Autodeclarada dos Participantes

\begin{tabular}{|c|c|c|c|}
\hline & Distribuição estatística & & \\
\hline Características & Grupos & $n$ & Percentagem \\
\hline \multirow[t]{4}{*}{ Gênero } & Masculino & 627 & $59,8 \%$ \\
\hline & Feminino & 410 & $39,1 \%$ \\
\hline & Travesti & 1 & $0,1 \%$ \\
\hline & Não binário & 11 & $1,0 \%$ \\
\hline \multirow[t]{5}{*}{$\begin{array}{l}\text { Orientação } \\
\text { Sexual }\end{array}$} & Heterossexual & 652 & $62,2 \%$ \\
\hline & Homossexual & 217 & $20,7 \%$ \\
\hline & Bissexual & 154 & $14,7 \%$ \\
\hline & Assexual & 7 & $0,7 \%$ \\
\hline & Pansexual & 19 & $1,8 \%$ \\
\hline \multirow[t]{6}{*}{$\begin{array}{l}\text { Status de } \\
\text { relacionamento }\end{array}$} & Solteiro(a) & 456 & $43,5 \%$ \\
\hline & Namorando & 343 & $32,7 \%$ \\
\hline & Casado(a) & 165 & $15,7 \%$ \\
\hline & Ficando & 71 & $6,8 \%$ \\
\hline & Viúvo(a) & 1 & $0,1 \%$ \\
\hline & Outros & 13 & $1,2 \%$ \\
\hline
\end{tabular}




\begin{tabular}{llrc}
\hline $\begin{array}{l}\text { Identificação } \\
\text { étnico-racial }\end{array}$ & Branco & 528 & $50,3 \%$ \\
\hline & Preto & 128 & $12,2 \%$ \\
\hline & Pardo & 365 & $34,8 \%$ \\
\hline & Amarelo & 18 & $1,7 \%$ \\
\hline Indígena & 6 & $0,6 \%$ \\
\hline menda domiciliar & Outros & 4 & $0,4 \%$ \\
\hline $\begin{array}{l}\text { Igual ou superior a 20 } \\
\text { salários mínimos (A) }\end{array}$ & 68 & $6,5 \%$ \\
\hline $\begin{array}{l}\text { De 10 a 20 salários } \\
\text { mínimos (B) }\end{array}$ & 110 & $10,5 \%$ \\
\hline $\begin{array}{l}\text { De 4 a 10 salários } \\
\text { mínimos (C) }\end{array}$ & 271 & $25,8 \%$ \\
\hline $\begin{array}{l}\text { De 2 a 4 salários } \\
\text { mínimos (D) }\end{array}$ & 266 & $25,4 \%$ \\
\hline $\begin{array}{l}\text { De até 2 salários } \\
\text { mínimos (E) }\end{array}$ & 224 & $21,4 \%$ \\
\hline $\begin{array}{l}\text { Inferior a 1 salário } \\
\text { mínimo (E-) }\end{array}$ & 110 & $10,5 \%$ \\
\hline
\end{tabular}

\section{Instrumentos}

Questionário Socioidentitário: foi criado pelos autores do artigo, composto por 19 questões centrais para os objetivos do estudo, esse questionário buscava caracterizar os participantes em diversas categorias (estado, idade, idade da primeira relação sexual, cor, escolaridade, relacionamento, nível socioeconômico, orientação sexual, gênero, percepção de afeminação e de masculinidade, entre outros).

Escala de Homofobia Internalizada - EHI (Lira \& Morais, 2019): Contém duas dimensões: Percepções internas do estigma e Percepções da opressão social. Apenas a primeira dimensão foi utilizada (15 itens), compondo o indicador de homofobia internalizada. Essa escala é composta, no total, por 19 itens e possui alfa de Cronbach de 0,814 e 0,622 respectivamente. É respondida através de uma escala do tipo Likert, de 4 pontos, que vai de " 1 - discordo totalmente" a " 4 - concordo totalmente". O escore foi calculado pela média aritmética.

Indicador de Violência Sofrida: criado por um dos autores para este estudo, é direcionado para indivíduos não-heterossexuais e objetiva mensurar a vivência de violência por conta da orientação sexual em 9 
modalidades de violência (e. g. física, psicológica, institucional). É respondida através de uma escala do tipo Likert, de 5 pontos, que vai de "0 - nunca sofri" a " 3 - aconteceu muitas vezes". O escore é calculado pela média aritmética.

Escala de Ansiedade em Interação Social (SIAS-6) e Escala de Ansiedade Social (SPS-6) (Ramos \& Cerqueira-Santos, 2021): compostas por 6 itens cada, avaliam ansiedade em interações sociais e ansiedade social diante da possibilidade de escrutínio por outras pessoas. Possuem alfa de Cronbach de 0,836 e 0,868, respectivamente. Juntas, compõem o indicador de ansiedade social deste estudo, com alfa de Cronbach de 0,902 . São respondidas através de uma escala do tipo Likert, de 5 pontos, que vai de " 1 - discordo fortemente" a " 5 - concordo fortemente". $\mathrm{O}$ escore foi calculado pela média aritmética.

\section{Procedimentos}

Os participantes foram informados dos procedimentos e objetivos da pesquisa através de um Termo de Consentimento Livre e Esclarecido e só participaram da aplicação do questionário os que declararam concordar com os termos da pesquisa e eram maiores de 18 anos. Os aspectos éticos que sustentam a integridade dos participantes foram garantidos com base na Resolução CNS 466/12 e 512/16. A pesquisa foi aprovada por um Comitê de Ética em Pesquisa.

\section{Análise de dados}

Para a realização das análises, foi utilizado o SPSS, versão 25. Foram realizadas análises estatísticas descritivas (levantamento de frequências, médias, desvios-padrão e amplitude). Assim como testes de inferência para as variáveis de interesse no estudo: ansiedade social relacionada com fatores característicos de minorias, como gênero, orientação sexual, homofobia internalizada e violência sofrida (teste $t$ de Student, ANOVA e regressão linear múltipla).

Para a análise da variável da EHI, foram criadas duas categorias: os com mais ou menos pontuação na escala, utilizou-se a média como ponto de corte. Além disso, também foram criados dois outros grupos para análise da violência sofrida: os com mais ou menos relatos de violência por conta da orientação sexual. Também se utilizou a média para discriminar os grupos. 


\section{Resultados}

Para investigar a influência da variável renda nos níveis de ansiedade social, foi executada uma comparação de médias por ANOVA. O efeito da renda nos níveis de ansiedade social resultou em diferenças significativas $[\mathrm{F}(5,1043)=5,987 ; \mathrm{p}<0,001]$. Os grupos com menores rendas (E e E-) apresentaram maiores médias de ansiedade social comparada aos demais, grupo $\mathrm{E}(\mathrm{M}=2,59 ; \mathrm{DP}=0,94)$ e grupo $\mathrm{E}-(\mathrm{M}=2,71$; $\mathrm{DP}$ $=0,97)$, conforme indicado na Figura 1 . A classe de menor renda (E-) diferiu significativamente das classes A ( $p=0,001), B(p=0,004)$ e C ( $p$ $=0,033)$, já a classe E somente da A $(p=0,006)$ e B $(p=0,019)$. Quando investigada a renda em consonância com o gênero, as mesmas proporções foram mantidas, mulheres de classes sociais mais baixas demonstraram maiores médias de ansiedade social comparadas aos homens da mesma renda, conforme a Figura 2.

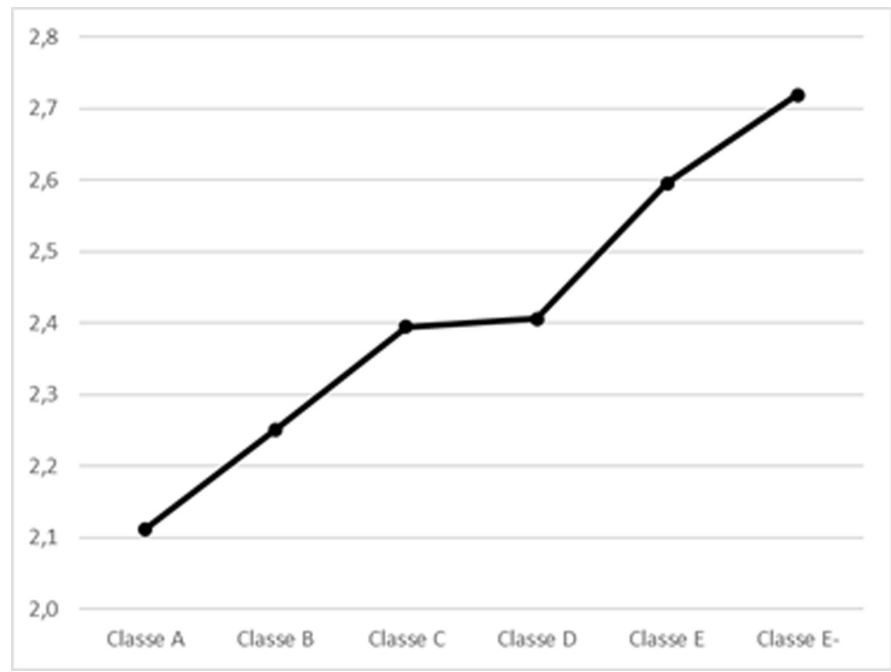

Figura 1: Média de ansiedade social por renda domiciliar 


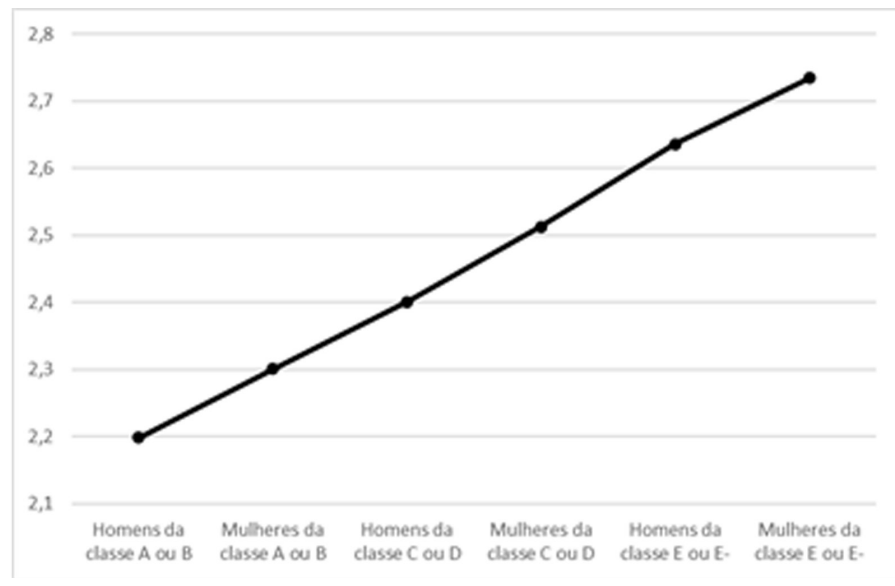

Figura 2: Inter-relação gênero e renda domiciliar com médias de ansiedade social

Com relação à influência da cor de pele $(\mathrm{n}=1021)$ nas médias de ansiedade, foi realizada outra ANOVA. Contudo, não houve diferença significativa em nenhuma das escalas $(p=0,702)$. $O$ total de participantes dessa análise foi reduzido para três categorias: branco, pardo e preto devido ao baixo número dos outros grupos presentes na amostra $(\mathrm{n}=28)$.

Para mensurar a diferença nos níveis de ansiedade social entre os gêneros, foi realizado um Teste t entre essa variável e a ansiedade social. Foram excluídos dessa análise os declarados não binários e travestis, devido ao baixo número de participantes presentes $(\mathrm{n}=12)$. $\mathrm{O}$ resultado encontrado apontou para a existência de diferença significativa $(\mathrm{t}=-3,780 ; \mathrm{p}<0,001)$. Entre os participantes dessa análise, as mulheres $(\mathrm{M}=2,56 ; \mathrm{DP}=0,90)$ apresentaram maiores médias contrapostas aos homens $(\mathrm{M}=2,34, \mathrm{DP}=0,94)$.

Foi realizada uma análise de variância unidirecional (ANOVA) entre os grupos de orientação sexual e a média de ansiedade social. A variável da orientação sexual foi recategorizada entre Heterossexuais, Homossexuais e Bissexuais ( $\mathrm{n}=1023)$. As outras orientações sexuais ( $\mathrm{n}$ = 26) foram excluídas da análise em cerne, devido ao baixo número de participantes. O resultado foi significativo $[\mathrm{F}(2,1020)=6,619 ; \mathrm{p}<$ $0,001]$. Por meio do teste post-hoc foi demonstrado que os bissexuais $(\mathrm{M}=2,62 ; \mathrm{DP}=0,96)$ diferem significativamente dos heterossexuais $(\mathrm{M}$ 
$=2,34 ; \mathrm{DP}=0,89)$. Os homossexuais $(\mathrm{M}=2,47 ; \mathrm{DP}=0,98)$ não diferiram significativamente dos heterossexuais, nem dos bissexuais - posicionando-se entre essas categorias.

Buscando-se analisar o gênero e a orientação inter-relacionados com as médias de ansiedade social foi realizada outra ANOVA. Participaram dessa análise os indivíduos dos gêneros feminino e masculino ( $\mathrm{n}=1037)$, formando assim quatro grupos: Mulheres heterossexuais, mulheres não-heterossexuais, homens heterossexuais e homens nãoheterossexuais. As análises indicaram diferença significativa $[\mathrm{F}(3,1027)$ $=10,830 ; \mathrm{p}<0,001]$. As mulheres não-heterossexuais $(\mathrm{M}=2,80 ; \mathrm{DP}=$ $0,90)$ apresentaram maiores médias de ansiedade social, conforme assinalado na Figura 3. Essas diferiram significativamente de todos os demais grupos em análise: homens heterossexuais $(\mathrm{M}=2,27$; DP $=0,90 ; \mathrm{p}<0,001)$, homens não-heterossexuais $(\mathrm{M}=2,42 ; \mathrm{DP}=1,00$; $\mathrm{p}=0,001)$ e mulheres heterossexuais $(\mathrm{M}=2,43 ; \mathrm{DP}=0,86 ; \mathrm{p}=0,001)$. Os outros grupos não diferiram entre si, apesar dos homens heterossexuais terem obtidos as menores médias de ansiedade social.

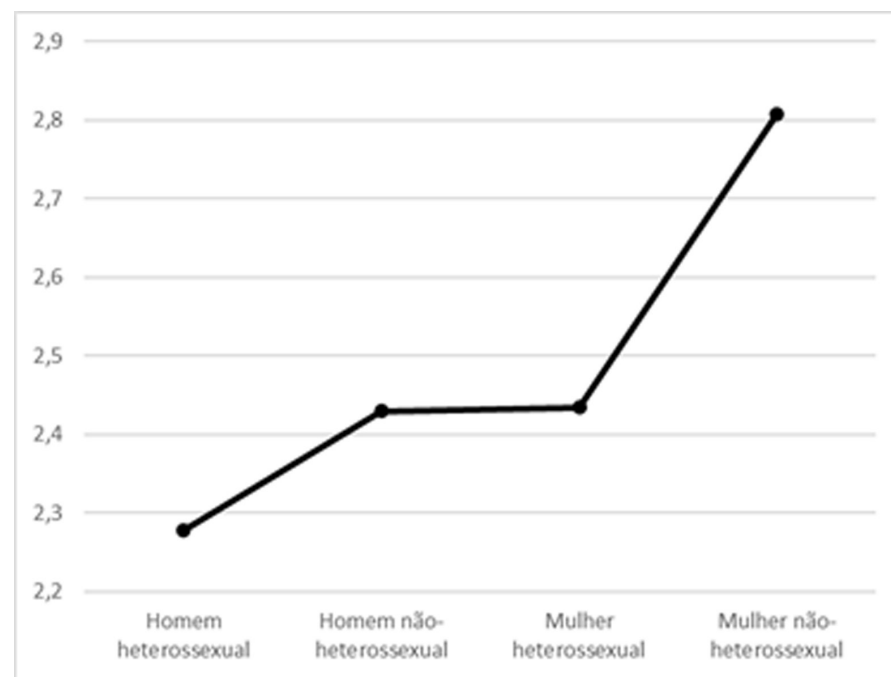

Figura 3: Inter-relação gênero e orientação sexual com média de ansiedade social

\section{Orientação Sexual e Ansiedade Social}

Partiu-se então para as análises direcionadas às pessoas não-heterossexuais. Participaram os indivíduos autodeclarados homossexuais e 
bissexuais $(n=371)$. Para avaliar a relação entre a homofobia internalizada (HI) e as médias de escalas de ansiedade foi realizado um novo Teste $\mathrm{t}$, os participantes foram divididos em dois grupos: os com maior pontuação de HI e os com menor pontuação de HI. O grupo de indivíduos que apresentou maior $\mathrm{HI}$ foi o que obteve maiores escores de ansiedade social $(\mathrm{t}=-2,644 ; \mathrm{p}=0,009)$. A média da ansiedade social para o grupo com menor $\mathrm{HI}$ foi de $2,45(\mathrm{DP}=0,98)$, enquanto para os com maiores níveis foi de 2,72 ( $\mathrm{DP}=1,00)$.

Posteriormente, foi analisada a relação dos grupos com mais e menos violência sofrida por conta da orientação sexual relatada com as médias de ansiedade social por meio de outro Teste t. Os resultados não demonstraram diferença significativa $(\mathrm{t}=-0,845 ; \mathrm{p}=0,399)$. O gênero quando posto em análise na violência sofrida $(\mathrm{t}=6,109 ; \mathrm{p}<0,001)$ demonstrou significativamente maior média para os homens $(M=0,74$; $\mathrm{DP}=0,52)$ em comparação às mulheres $(\mathrm{M}=0,42 ; \mathrm{DP}=0,43)$. Outro Teste $t$ foi executado para analisar a influência da variável afeminação ( $n=248$ ) nos níveis de violência sofrida pelos homens não-heterossexuais, demonstrando resultado significativo $(\mathrm{t}=5,669 ; \mathrm{p}<0,001)$. Os autodeclarados como afeminados $(\mathrm{M}=0,98$; $\mathrm{DP}=0,51)$ apresentaram maiores médias de violência sofrida em relação aos não-afeminados $(\mathrm{M}=0,601 ; \mathrm{DP}=0,48)$. O mesmo foi feito para averiguar a relação da afeminação com a ansiedade social, a diferença não foi significativa ( $\mathrm{t}$ $=1,665 ; \mathrm{p}=0,96)$.

Duas regressões lineares múltiplas foram realizadas, com método Backward, para investigar se a ansiedade social poderia ser predita por variáveis pertinentes para a configuração do modelo estresse de minoria: gênero, idade, renda, HI e violência sofrida. Novamente, a amostra utilizada foi composta apenas pelos homossexuais e bissexuais. O modelo apresentou resultado significativo $[\mathrm{F}(5,365)=13,706 ; \mathrm{p}<0,001$; $\left.r^{2}=0,193\right]$; sendo o gênero $(\beta=0,176 ; t=3,509, p=0,001)$, a idade $(\beta$ $=-0,214 ; \mathrm{t}=-4,349 ; \mathrm{p}<0,001)$, a média de violência sofrida $(\beta=0,116$; $\mathrm{t}=2,356 ; \mathrm{p}=0,019)$, a média de HI $(\beta=0,222 ; \mathrm{t}=4,594 ; \mathrm{p}<0,001)$ e a renda $(\beta=0,184 ; \mathrm{t}=3,811 ; \mathrm{p}<0,001)$ preditores de ansiedade social. Os resultados sugeriram que ter menos idade e pertencer a grupos sujeitos ao estresse minoritário, como ser mulher, ter menor renda, mais homofobia internalizada e sofrer mais violência, estão relacionados com indicadores de ansiedade social, sendo, portanto, fatores de risco. 


\section{Discussão}

O estudo evidenciou que pertencer a um grupo minoritário atua como um importante fator associado a sintomas de ansiedade social, assim como a sobreposição entre identidades minoritárias diferentes repercutem em um maior acúmulo e experimentação de estigmas sociais, e por sua vez, em uma maior possibilidade de desfechos negativos em saúde mental. Além disso, demonstrou-se que em minorias sexuais, a presença de homofobia internalizada está relacionada a maiores níveis de indicativos de ansiedade social. Assim como, a associação de estereótipos femininos em figuras masculinas incide em uma maior percepção e experimentação de violência sofrida nesses indivíduos.

Esses resultados denotam como a exposição a estigmas, preconceitos e eventos discriminatórios podem atuar como fator de risco para a incidência de transtornos mentais. Nessa direção, as políticas orientadas para o cuidado da população necessitam estar em consonância com o efeito dos estressores sociais, como o pertencimento a grupos minoritários.

O gênero apresentou relação direta com os níveis de ansiedade social encontrados. Mulheres apresentaram maiores médias nos indicativos de ansiedade social quando comparadas aos homens. Tais diferenças são corroboradas pela literatura (Asher, Asnaani, \& Aderka, 2017), posto que mulheres são socializadas a internalizar mais estresse em comparação aos homens, fator que repercute na incidência de psicopatologias juntamente com os estressores específicos experimentados por esse grupo.

Grupos com rendas menores apresentaram maior prevalência de ansiedade. As disparidades econômicas estão relacionadas a sensações de humilhação, inferioridade, ausência de controle sobre a circunstância vivenciada e insuficiência (Ludermir, 2008). Fatores como o desemprego e o índice de desigualdade de renda também acentuam a experimentação de ansiedade nessa população (Rocha et al., 2015). Em sua maior parte, indivíduos de baixa renda dependem de sistemas de saúde públicos. Assim, discrepâncias orçamentárias na manutenção de serviços em saúde mental, como capacitação e número de profissionais por indivíduo, interferem na assistência psicológica dessa população (Salvador-Carulla, Garrido, McDaid, \& Haro, 2006). Não 
obstante, crises econômicas afetam de modo significativo esse sistema, gerando redução de investimentos no setor, e por consequência maior precarização no manejo da saúde mental (Rocha et al., 2015).

Pertencer a grupos de baixa renda e possuir menor escolaridade está relacionado a uma maior incidência de psicopatologias (Rocha et al., 2015), principalmente em mulheres (Rocha et al., 2010). Por conseguinte, mulheres de classes sociais mais baixas detém menor capacidade de recursos econômicos, e por sua vez, menor possibilidade de dispor de auxílio nas tarefas domésticas (Artazcoz, Borrell, Benach, Cortès, \& Rohlfs, 2004). Destarte, tendem a ter menor conciliação entre a vida profissional e familiar, sendo mais vulneráveis a desfechos negativos em saúde mental (Artazcoz et al., 2004).

Na revisão de literatura feita por Smolen e Araújo (2017) foi encontrada uma prevalência de transtornos mentais em populações não-brancas. Resposta que pode ser explicada pelo fenômeno do racismo, ou seja, a crença na superioridade de uma raça em detrimento de outra, que atua como uma forma de violência sobre a saúde mental dos que o sofrem (Damasceno \& Zanello, 2018). Contudo, no presente estudo não houve diferença estatística nesse dado; ainda que, no Brasil, exista um forte estigma ao se identificar como negro, havendo uma tentativa de embranquecimento dessa população, a negação do ser negro (Veiga, 2019).

Lésbicas, gays e bissexuais demonstraram maiores indicadores de ansiedade social contrapostos a indivíduos heterossexuais, com destaque para as mulheres não-heterossexuais. Há uma maior prevalência de desfechos negativos na saúde mental desse grupo (Pakula, Shoveller, Ratner, \& Carpiano, 2016; Paveltchuk \& Borsa, 2019) devido à experimentação do estresse minoritário que estão submetidos (Herek et al., 2009; Meyer, 2003); isto é, a exposição a estigmas, à rejeição e aos eventos discriminatórios dentro de uma sociedade heterossexista (Meyer, 1995). Pode-se afirmar que caso o indivíduo faça parte de um grupo minoritário, os conflitos oriundos do convívio social tendem a apresentar maior dano, bem como maior efeito de estresse (Meyer, 1995).

Fatores como baixa rede de apoio, discriminação social e homofobia internalizada são algumas das causas para essa prevalência (Pakula et al., 2016). Os indivíduos que apresentaram maiores escores de 
homofobia internalizada também demonstraram maiores médias de ansiedade social. $\mathrm{O}$ estigma quando internalizado pode resultar em sentimentos aversivos, como sofrimento emocional, baixa autoestima e abuso de substâncias (Antunes, 2017; Lehavot \& Simoni, 2011).

Ao comparar lésbicas, gays e bissexuais em níveis de ansiedades, os autodeclarados como bissexuais apresentaram maiores médias. Indivíduos bissexuais podem demonstrar pior estado de saúde mental quando comparados a outras minorias sexuais (Feinstein \& Dyar, 2017; Pakula et al., 2016). A bifobia predispõe os bissexuais a apresentarem um comportamento de identidade sexual mais ambíguo, e, por sua vez, menor oportunidade de usufruir de recursos sociais de manejo e/ou suporte dentro e fora da comunidade não-heterossexual (Dyar, Feinstein, \& London, 2015; la Roi, Meyer, \& Frost, 2019; Paveltchuk \& Borsa, 2019).

Os transtornos psicológicos são mais díspares quando há a análise do gênero nas minorias sexuais. Quando há a inter-relação entre identidades marginalizadas, há um acúmulo de estigmas, e uma maior disparidade na incidência de transtornos. $\mathrm{O}$ estresse minoritário vivido, com destaque por mulheres bissexuais, pode resultar em incerteza sobre a identidade sexual (Dyar et al., 2015). Pois, são, com frequência, consideradas lésbicas perante o social e presenciam sua identidade bissexual de antemão negada (Dyar et al., 2015). Essa minoria vivencia menor processo de abertura da orientação sexual para as suas famílias (Dyar et al., 2015), por temerem maior rejeição e discriminação, desfrutando de menores redes de apoio. Sendo o processo de abertura um importante preditor de saúde mental (Kosciw, Palmer, \& Kull, 2015). Entretanto, especificamente para bissexuais há evidências contraditórias sobre os efeitos positivos da abertura da orientação sexual (Feinstein et al., 2019).

Neste estudo, os homens não-heterossexuais demonstraram sofrer mais violência por conta de sua orientação sexual do que as mulheres não-heterossexuais, fenômeno já conhecido na literatura (Ortiz-Hernández \& Cosme, 2006). Os homens autoidentificados como afeminados experimentaram maior nível de violência, essa poderia ser uma das motivações para esses episódios de violência. Dentro das construções hierárquicas, os homens são socializados para exercerem força e assertividade. Conforme Ramos e Cerqueira-Santos (2020), quanto mais próximo de um modelo hegemônico e idealizado de masculini- 
dade, maior a concessão de poder. Diante disso, os que se aproximam da feminilidade desfrutam de menos benefícios sociais. Não por acaso, a violência aos afeminados compartilham incentivos existentes aos direcionados às mulheres (Ramos \& Cerqueira-Santos, 2020).

Pertencer a grupos estigmatizados, seja de gênero, classe, raça ou orientação sexual parece estar relacionado a maiores níveis de violência individual e sujeição a eventos estressores. Uma maior idade atua como fator protetivo no desenvolvimento do transtorno de ansiedade, havendo maior incidência no início da adolescência. Conforme o Manual Diagnóstico e Estatístico de Transtornos Mentais - DSM-5 (American Psychiatric Association, 2014), casos em adultos mais velhos ocorrem em menor proporção. Esses podem decorrer após a experimentação de uma situação estressora e/ou humilhante ou após alterações que exijam desempenhos de novas funções sociais (American Psychiatric Association, 2014).

Este trabalho apresenta limitações: a pesquisa foi realizada por plataformas virtuais, o que não garante a representatividade de uma parte da população, já que há uma parcela significativa que não dispõe de internet no Brasil. Esta foi a primeira pesquisa na qual o indicador de violência sofrida foi utilizado, o que poderia prejudicar a confiabilidade da medida. Além disso, a maioria dos respondentes possuía alto nível de escolaridade, o que leva a um viés de resposta, considerando a influência da educação na relação com os estigmas.

É urgente a análise dos marcadores do estresse social para a compreensão da saúde mental em grupos minoritários. Assim, pesquisas futuras devem examinar as divergências experimentadas de maneira mais específicas, para sustentar uma melhor análise dos múltiplos estressores e abarcar os possíveis efeitos de suas intersecções, analisando, por exemplo, os recortes de gênero inferidos em outras minorias hierárquicas. Assim, conhecendo as disparidades enfrentadas, pode haver uma atuação mais eficaz na saúde mental desses grupos.

\section{Referências}

Alves, R. Á. K., Paveltchuk, F. D. O., Carvalho, M. R. D., \& Falcone, E. M. D. O. (2017). Alterando crenças centrais: um relato de caso de homofobia internalizada. Revista Brasileira de Terapias Cognitivas, 13(1), 12-19. DOI: 10.5935/1808-5687.20170004 
American Psychiatric Association. (2014). DSM-5: Manual diagnóstico e estatístico de transtornos mentais. Artmed Editora

Antunes, P. P. S. (2017). Homofobia internalizada: o preconceito do homossexual contra si mesmo. Annablume.

Artazcoz, L., Borrell, C., Benach, J., Cortès, I., \& Rohlfs, I. (2004). Women, family demands and health: the importance of employment status and socio-economic position. Social science \& medicine, 59(2), 263-274. DOI: 10.1016/j.socscimed.2003.10.029

Assari, S. (2019). Race, depression, and financial distress in a nationally representative sample of American adults. Brain sciences, 9(2), 29.

Asher, M., Asnaani, A., \& Aderka, I. M. (2017). Gender differences in social anxiety disorder: A review. Clinical psychology review, 56, 1-12. DOI: 10.1016/j.cpr.2017.05.004

Bilodeau, J., Marchand, A., \& Demers, A. (2020). Psychological distress inequality between employed men and women: A gendered exposure model. SSM-population health, 11, 100626.

Cohen, J. M., Blasey, C., Taylor, C. B., Weiss, B. J., \& Newman, M. G. (2016). Anxiety and related disorders and concealment in sexual minority young adults. Behavior Therapy, 47(1), 91101. DOI: 10.1016/j.beth.2015.09.006

Connor, R. A., Glick, P., \& Fiske, S. T. (2016). Ambivalent sexism in the twenty-first century. In Sibley, C. G., Barlow, F. K. (Eds.), The Cambridge handbook of the psychology of prejudice (pp. 295320). Cambridge, MA: Cambridge University Press.

Damasceno, M. G., \& Zanello, V. M. L. (2018). Saúde mental e racismo contra negros: produção bibliográfica brasileira dos últimos quinze anos. Psicologia: Ciência e Profissão, 38(3), 450-464

Dyar, C., Feinstein, B. A., \& London, B. (2015). Mediators of differences between lesbians and bisexual women in sexual identity and minority stress. Psychology of Sexual Orientation and Gender Diversity, 2(1), 43.

Duarte, D., \& Paulino, P. R. V. (2021). O machismo e sua influência nas crenças centrais femininas. Cadernos de psicologia, 2(4).

Goto, J. B., Couto, P. F. M., \& Bastos, J. L. (2013). Revisão sistemática dos estudos epidemiológicos sobre discriminação interpessoal e saúde mental. Cadernos de Saúde Pública, 29, 445-459. 
Feinstein, B. A., \& Dyar, C. (2017). Bisexuality, minority stress, and health. Current Sexual Health Reports, 9(1), 42-49. DOI: 10.1007/s11930-017-0096-3

Feinstein, B. A., Dyar, C., Li, D. H., Whitton, S. W., Newcomb, M. E., \& Mustanski, B. (2019). The longitudinal associations between outness and health outcomes among gay/lesbian versus bisexual emerging adults. Archives of sexual behavior, 48(4), 1111-1126.

Frost, D. M., Lehavot, K., \& Meyer, I. H. (2015). Minority stress and physical health among sexual minority individuals. Journal of behavioral medicine, 38(1), 1-8. DOI: 10.1007/s10865-0139523-8

Frost, D. M., Meyer, I. H. (2012). Measuring community connectedness among diverse sexual minority populations. Journal of sex research, 49(1), 36-40. DOI: 10.1080/00224499.2011.565427

Hendricks, M. L., \& Testa, R. J. (2012). A conceptual framework for clinical work with transgender and gender nonconforming clients: An adaptation of the Minority Stress Model. Professional Psychology: Research and Practice, 43(5), 460.

Herek, G. M., Cogan, J. C., Gillis, J. R., \& Glunt, E. K. (1998). Correlates of internalized homophobia in a community sample of lesbians and gay men. Journal-Gay and Lesbian Medical Association, 2, 17-26.

Herek, G. M., Gillis, J. R., \& Cogan, J. C. (2009). Internalized stigma among sexual minority adults: Insights from a social psychological perspective. Journal of Counseling Psychology, 56(1), 32. DOI: $10.1037 / \mathrm{a} 0014672$

Juárez-Chávez, E., Cooney, E. E., Hidalgo, A., Sánchez, J., \& Poteat, T. (2018). Violence experiences in childhood and adolescence among gay men and transgender women living in Peru: a qualitative exploration. Journal of interpersonal violence, 0886260518787811.

Koh, A. S., \& Ross, L. K. (2006). Mental health issues: A comparison of lesbian, bisexual and heterosexual women. Journal of homosexuality, 51(1), 33-57.

Kosciw, J. G., Palmer, N. A., \& Kull, R. M. (2015). Reflecting resiliency: Openness about sexual orientation and/or gender identity and its relationship to well-being and educational outcomes for LGBT students. American journal of community psychology, 55(1-2), 167-178. DOI: 10.1007/s10464-014-9642-6 
La Roi, C., Meyer, I. H., \& Frost, D. M. (2019). Differences in sexual identity dimensions between bisexual and other sexual minority individuals: Implications for minority stress and mental health. American Journal of Orthopsychiatry, 89(1), 40. DOI: $10.1037 /$ ort0000369

Lefevor, G. T., Janis, R. A., Franklin, A., \& Stone, W. M. (2019). Distress and therapeutic outcomes among transgender and gender nonconforming people of color. The Counseling Psychologist, 47(1), 34-58.

Lehavot, K., \& Simoni, J. M. (2011). The impact of minority stress on mental health and substance use among sexual minority women. Journal of consulting and clinical psychology, 79(2), 159. doi:10.1037/a0022839

Lira, A. N. D., \& Morais, N. A. D. (2019). Validity Evidences of the Internalized Homophobia Scale for Brazilian Gays and Lesbians. Psico-USF, 24(2), 361-372. DOI: 10.1590/141382712019240212

Ludermir, A. B. (2008). Desigualdades de classe e gênero e saúde mental nas cidades. Physis: Revista de Saúde Coletiva, 18(3), 451467. DOI: $10.1590 /$ S0103-73312008000300005

McClain, S., Beasley, S. T., Jones, B., Awosogba, O., Jackson, S., \& Cokley, K. (2016). An examination of the impact of racial and ethnic identity, impostor feelings, and minority status stress on the mental health of Black college students. Journal of Multicultural Counseling and Development, 44(2), 101-117.

Meyer, I. H. (1995). Minority stress and mental health in gay men. Journal of health and social behavior, 38-56. doi:10.2307/2137286

Meyer, I. H. (2003). Prejudice, social stress, and mental health in lesbian, gay, and bisexual populations: Conceptual issues and research evidence. Psychological Bulletin, 129, 674 - 697.

Meyer, I. H., \& Frost, D. M. (2013). Minority stress and the health of sexual minorities. In C. J. Patterson \& A. R. D'Augelli (Eds.), Handbook of psychology and sexual orientation (p. 252-266). Oxford University Press.

Newcomb, M. E., \& Mustanski, B. (2010). Internalized homophobia and internalizing mental health problems: A meta-analytic review. Clinical psychology review, 30(8), 1019-1029. DOI: 10.1016/j.cpr.2010.07.003 
Ortiz-Hernández, L., \& Granados-Cosme, J. A. (2006). Violence against bisexuals, gays and lesbians in Mexico City. Journal of Homosexuality, 50(4), 113-140. DOI: 10.1300/J082v50n04_06

Pachankis, J. E., \& Goldfried, M. R. (2006). Social anxiety in young gay men. Journal of anxiety disorders, 20(8), 996-1015. DOI: 10.1016/j.janxdis.2006.01.001

Pakula, B., Shoveller, J., Ratner, P. A., \& Carpiano, R. (2016). Prevalence and co-occurrence of heavy drinking and anxiety and mood disorders among gay, lesbian, bisexual, and heterosexual Canadians. American journal of public health, 106(6), 10421048. DOI: 10.2105/AJPH.2016.303083

Paveltchuk, F. O., \& Borsa, J. C. (2019). Homofobia internalizada, conectividad comunitaria y salud mental en una muestra de individuos LGB brasileños. Avances en psicología latinoamericana, 37(1), 47-61.

Paveltchuk, F. O., \& Borsa, J. C. (2020). A teoria do estresse de minoria em lésbicas, gays e bissexuais. Revista da SPAGESP, 21(2), 41-54.

Ramos, M. de M., \& Cerqueira-Santos, E. (2020). Afeminação, hipermasculinidade e hierarquia. Arquivos Brasileiros de Psicologia, 72(1), 159-172.

Ramos, M. de M., \& Cerqueira-Santos, E. (2021). Ansiedade Social: adaptação e evidências de validade da forma curta da Social Interaction Anxiety Scale e da Social Phobia Scale para o Brasil. Jornal Brasileiro de Psiquiatria, 70(2), 149-156.

Riggle, E. D. B., Folberg, A. M., Richardson, M. T., \& Rostosky, S. S. (2021). A measure of hypervigilance in LGBTQ-identified individuals. Stigma and Health. Advance online publication.

Rocha, K. B., Pérez, K., Rodríguez-Sanz, M., Borrell, C., \& Obiols, J. E. (2010). Prevalencia de problemas de salud mental y su asociación con variables socioeconómicas, de trabajo y salud: resultados de la Encuesta Nacional de Salud de España. Psicothema, 22(3), 389-395.

Rocha, K. B., Perez, K., Rodriguez-Sanz, M., Muntaner, C., Alonso, J., \& Borrell, C. (2015). Inequalities in mental health in the spanish autonomous communities: a multilevel study. The Spanish journal of psychology, 18. doi:10.1017/sjp.2015.28

Salvador-Carulla, L., Garrido, M., McDaid, D., \& Haro, J. M. (2006). Financing mental health care in Spain: context and critical issues. The European journal of psychiatry, 20(1), 29-44. 
Schwartz, S., \& Meyer, I. H. (2010). Mental health disparities research: The impact of within and between group analyses on tests of social stress hypotheses. Social Science and Medicine, 70, 1111-1118. DOI: 10.1016/j.socscimed.2009.11.032

Silva, D. F., \& de Santana, P. R. (2012). Transtornos mentais e pobreza no Brasil: uma revisão sistemática. Tempus Actas de Saúde Coletiva, 6(4), 175-185. DOI: 10.18569/tempus.v6i4.1214

Silveira, A. P., \& Cerqueira-Santos, E. (2019). Homofobia internalizada e satisfação sexual em casais homossexuais. Psicogente, 22(41), 1-18. doi:10.17081/psico.22.41.3307

Smolen, J. R., \& Araújo, E. M. D. (2017). Raça/cor da pele e transtornos mentais no Brasil: uma revisão sistemática. Ciência \& Saúde Coletiva, 22, 4021-4030.

Veiga, L. M. (2019). Descolonizando a psicologia: notas para uma Psicologia Preta. Fractal: Revista de Psicologia, 31(SPE), 244248. DOI: 10.22409/1984-0292/v31i_esp/29000

Wadsworth, L. P., \& Hayes-Skelton, S. A. (2015). Differences among lesbian, gay, bisexual, and heterosexual individuals and those who reported an other identity on an open-ended response on levels of social anxiety. Psychology of sexual orientation and gender diversity, 2(2), 181.

Walch, S. E., Ngamake, S. T., Bovornusvakool, W., \& Walker, S. V. (2016). Discrimination, internalized homophobia, and concealment in sexual minority physical and mental health. Psychology of Sexual Orientation and Gender Diversity, 3(1), 37. DOI: $10.1037 /$ sgd0000146 